Disclosure of Interest: None declared

DOI: 10.1136/annrheumdis-2018-eular.5341

\section{SAT0217 IDENTIFICATION OF DISTINCT DISEASE ACTIVITY TRAJECTORIES IN PATIENTS WITH RHEUMATOID ARTHRITIS RECEIVING TOFACITINIB OVER 12 MONTHS}

V. P. Bykerk ${ }^{1}$, R. van Vollenhoven ${ }^{2}$, C. Connell ${ }^{3}$, D. Gruben ${ }^{3}$, L. Fallon ${ }^{4}$, J. Woolcott ${ }^{5}$, E. Keystone ${ }^{6}$. ${ }^{1}$ Weill Cornell Medical College, New York, NY, United States, ${ }^{2}$ Amsterdam Rheumatology and Immunology Center, Amsterdam, Netherlands, ${ }^{3}$ Pfizer Inc, Groton, CT, United States, ${ }^{4}$ Pfizer Canada, Montreal, QC Canada, ${ }^{5}$ Pfizer Inc, Collegeville, PA, United States, ${ }^{6}$ Mount Sinai Hospital, Toronto, ON, Canada

Background: Persistence of active disease in patients (pts) with rheumatoid arthritis (RA) is highly variable following treatment initiation. One possible explanation is the existence of distinct disease activity/response trajectories influenced by baseline variables, such as sociodemographics, disease characteristics and health status. ${ }^{1}$

Objectives: To identify distinct disease activity trajectories over 12 months and distinguishing baseline factors using pooled data from 3 randomised, controlled Phase 3 (P3) studies of tofacitinib $5 \mathrm{mg}$ twice daily (BID) in pts with active RA who were inadequate responders (IR) to conventional synthetic (cs)DMARDs, with or without prior biologic (b)DMARDs (NCT00847613, NCT00856544 and NCT00853385).

Methods: Disease Activity Score in 28 joints, erythrocyte sedimentation rate (DAS28-4[ESR]) data from 3 P3 studies were pooled. A group-based trajectory modelling strategy was applied to find unique longitudinal groups of pts with similar disease activity over time. ${ }^{2}$ Trajectories were latent models, fit as polynomials. The number of groups and polynomial degree of each group were specified and fit for all combinations of up to 6 groups and up to a 4th degree polynomial; a best-fit model was chosen using Bayesian information criteria.

Results: csDMARD-IR/bDMARD-naïve pts $(n=677)$ were separated into 5 unique disease activity trajectories (figure 1); csDMARD-IR pts who received prior bDMARDs $(n=149)$ were separated into 4 trajectories (not shown). In the bDMARD-naïve pts, Group 5 (3.8\%) had the highest predicted baseline DAS (7.3) with minimal improvement (6.4) at Month 12; Group 4 (26.7\%) had baseline DAS 7.0, improving to DAS 5.0 at Month 12 ; Groups $2(30.4 \%)$ and $3(36.6 \%)$ had baseline DAS 5.9 and 6.2, respectively, leading to low DAS (2.9) and moderate DAS (3.9), respectively, at Month 12; Group 1 (2.4\%) had the lowest baseline DAS of 4.3, improving to remission (DAS 2.1) at Month 12 (figure 1). The 4 trajectories in cSDMARD-IR pts who received prior bDMARDs were similar to Groups $2-5$ in bDMARD-naïve pts. While baseline demographics were generally similar between bDMARD-naïve groups, there were statistically significant differences in baseline disease activity measures and pt-relevant outcomes between groups.

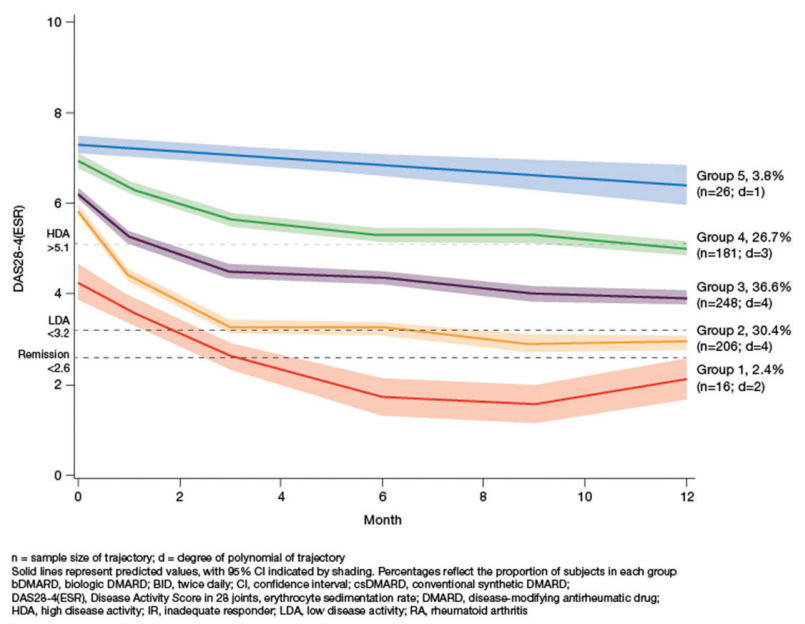

Figure 1. Predicted group trajectories based on DAS28-4(ESR) with $95 \% \mathrm{Cl}$ identified using group-based trajectory modeling in patients with active RA who were csDMARD-IR and bDMARD-naïve and receiving tofacitinib $5 \mathrm{mg} B I D n=$ sample size of trajectory; $d$ = degree of polynomial of trajectory

Conclusions: It was possible to identify heterogeneous phenotypic subgroups as distinct disease activity trajectories in cSDMARD-IR pts treated with tofacitinib.
The groups were characterised by differences in disease activity and pt-relevant outcomes, including baseline pain and physical function. Very high disease activity may limit pts' ability to achieve low disease activity. The identification of distinct trajectory groups could be used to develop personalised treatment optimisation algorithms incorporating clinical and molecular phenotypes.

\section{REFERENCES :}

[1] Barnabe C, et al. PLoS One 2015;10:e0135327.

[2] Nagin DS. In: Handbook of Quantitative Criminology. New York, NY Springer 2010:53-67.

Acknowledgements: Study sponsored by Pfizer Inc. Medical writing support was provided by R Knight of CMC and funded by Pfizer Inc.

Disclosure of Interest: V. Bykerk Grant/research support from: Amgen, BMS, Genentech, UCB, Consultant for: AbbVie, Amgen, BMS, Gilead, Pfizer Inc, Sanofi-Regeneron, UCB, R. van Vollenhoven Grant/research support from: AbbVie, Amgen, BMS, GSK, Pfizer Inc, Roche, UCB, Consultant for: AbbVie, AstraZe neca, Biotest, BMS, Celgene, Crescendo, Eli Lilly, GSK, Janssen, Merck Novartis, Pfizer Inc, Roche, UCB, Vertex, C. Connell Shareholder of: Pfizer Inc, Employee of: Pfizer Inc, D. Gruben Shareholder of: Pfizer Inc, Employee of: Pfize Inc, L. Fallon Shareholder of: Pfizer Inc, Employee of: Pfizer Inc, J. Woolcott Shareholder of: Pfizer Inc, Employee of: Pfizer Inc, E. Keystone Grant/research support from: AbbVie, Amgen, BMS, Eli Lilly, Pfizer Inc, Roche, Consultant for: AbbVie, Amgen, BMS, Eli Lilly, Pfizer Inc, Roche

DOI: 10.1136/annrheumdis-2018-eular.1281

\section{SAT0218 EFFICACY AND SAFETY OF BARICITINIB IN MTX-IR PATIENTS WITH RHEUMATOID ARTHRITIS: 52 WEEK RESULTS FROM A PHASE 3 STUDY (RA-BALANCE)}

Z. Li ${ }^{1}$, J. $\mathrm{Hu}^{2}$, C. Bao ${ }^{3}$, X. Li ${ }^{4}$, X. Li ${ }^{5}$, J. Xu' ${ }^{6}$ A. Spindler ${ }^{7}$, X. Zhang ${ }^{8}$, J. Xu$^{9}$, Z. Li ${ }^{10}$, G. Wang ${ }^{11}$, J. Sun ${ }^{12}$, F. Ji ${ }^{12}$, H. Tao ${ }^{12}$, L. Zhan ${ }^{12}$, T. Rooney $^{13}$, C. Zerbini ${ }^{14}$. ${ }^{1}$ Peking University People's Hospital, Beijing, ${ }^{2}$ Pingxiang People's Hospital, Pingxiang, ${ }^{3}$ Renji Hospi. Affliated to SH Jiaotong U, Shanghai, ${ }^{4}$ Qilu Hospital of Shandong University, Shandong, ${ }^{5}$ Anhui Provincial Hospital, ${ }^{6} 1$ st Affiliate Hospital of AnHui Med U, Anhui, China, ${ }^{7}$ Centro Medico Privado de Reumatologica, Tucuman, Argentina, ${ }^{8}$ Guangdong General Hospital, Guangdong, ${ }^{9} 1$ st Affiliated Hospital of Kunming Med U, Kunming, ${ }^{10} 1$ st Afflilated Hospital of Bengbu Med C, Anhui, ${ }^{11}$ China-Japan Friendship Hospital, Beijing, ${ }^{12}$ Eli Lilly and Company, Shanghai, China, ${ }^{13}$ Eli Lilly and Company, Indianapolis, United States, ${ }^{14}$ Centro Paulista de Investigacao Clinica, São Paulo, Brazil

Background: Baricitinib (BARI) is an oral selective inhibitor of Janus kinase 1 (JAK1) and JAK2. In the EU and some other countries, baricitinib has been approved for the treatment of moderately to severely active rheumatoid arthritis (RA) in adult patients. This abstract reports efficacy and safety results from a phase 3, double-blinded, 52-week study (RA-BALANCE) that enrolled patients (pts) in China, Argentina and Brazil (NCT02265705).

Objectives: To assess the efficacy and safety of BARI vs placebo (PBO) in the treatment of RA.

Methods: Patients with moderately to severely active RA (tender joint counts $\geq 6$ \& swollen joint counts $\geq 6$ \& hsCRP $\geq 6 \mathrm{mg} / \mathrm{L}$ ) despite stable background methotrexate (MTX), were randomized 1:1 to PBO $(n=145)$ or BARI 4-mg $(n=145)$ once daily (QD), stratified by country and baseline joint erosion status. Background MTX was continued. Non-responders were rescued from Week 16. At Week 24 pts receiving PBO were switched to BARI 4-mg QD. ACR20 at Week 12 was the primary endpoint and there were multiple secondary endpoints e.g., assessing physical function, low disease activity and pain.

Results: The primary ACR20 response was significantly greater for BARI than PBO (58.6\% vs $28.3 \%, \mathrm{p} \leq 0.001$, Table). At Weeks 12 and 24 , significant improvements were seen in pts receiving BARI vs PBO for ACR20/50/70, DAS28-hsCRP, CDAI low disease activity and SDAI low disease activity, many as early as by Week 1. At Week 16, significantly less radiographic progression was seen in pts receiving $B A R I$ vs $P B O$ and numerical improvement was observed at Week 24 . A Week 12, significant improvement in HAQ-DI minimum clinically important difference $\geq 0.3$ (physical function), duration of morning joint stiffness, severity of morning joint stiffness numeric rating scale (NRS), worst tiredness NRS and reduced pain (0-100 mm VAS) were seen in pts receiving BARI vs PBO.

During Weeks $0-24$, treatment emergent adverse events and infections were reported in $74.5 \%$ and $42.1 \%$ of BARI pts and $62.1 \%$ and $28.3 \%$ of PBO pts, respectively. Serious adverse events were reported in $2.8 \%$ of pts in both groups There was 1 nonserious esophageal candidiasis in the BARI group for Week 0 24. Four herpes zoster events (1 PBO, 3 BARI) were reported for Week 0-24. No major cardiovascular events, deaths, tuberculosis, venous thromboembolic 